\title{
The role of teachers in developing students' autonomous learning ability Maohua SUN
}

\author{
Foreign Language Department, Dalian Jiao Tong University, Liaoning Province, China
} summer817@163.com

Keywords: autonomous learning, teacher role

\begin{abstract}
Autonomous learning is influenced by many factors, such as teacher autonomy, learning strategies, learning styles and learning. Learning motivation, culture and society, and technical assistance. Among these factors, the role of teachers is more important. Because it is closely related to autonomous learning. In the practice of autonomous learning, teachers are the design participants of autonomous learning activities. Style and content directly affect whether students can make full and effective use of various resources for autonomous learning.
\end{abstract}

\section{浅析培养学生自主学习能力中教师的作用}

\author{
孙茂华 \\ 大连交通大学, 大连, 辽宁省, 中国 \\ summer_817@163.com
}

关键词:自主学习, 教师角色

中文摘要.自主学习受到许多因素的影响, 例如教师自主性、学习策略、学习方式、学习动机、 文化和社会、以及技术协助。在这些因素中，教师的作用相对更重要，因为它与自主学习密 切相关。在自主学习实践中, 教师对自主学习活动的设计方式与内容直接影响着学生能否充 分而有效地利用各种资源进行自主学习。

\section{1. 教师角色的转变}

教师要在培养学生自主学习能力中发挥作用, 就要实现角色的转变。在传统的教学模式 中, 教学以教师为主导, 以应试为目的, 主要讲授语法和词汇。评估教师的标准在于他们给 学生灌输多少知识, 自然是越多越好。教师是冊庸置疑的 “权威”, 一个 “给予知识的人”, 他们自始至终都支配着整个课堂。而学生只是 “无知的观众” , 记录下珍贵的词组, 还要为 此喝彩。这种教学重在强调语言能力, 而不是应用能力, 整个过程中教师和学生之间没有什 么合作, 而教师也很少能从他的 “观众” 中获得反馈意见。在自主学习的教学模式中, 教师和学生的角色都有所转变, 各自所扮演的角色也发生了变化。目前被普遍接受的在自主 学习过程中教师应扮演的有六大角色: 学习引导者(guide)、目标确定者 (go al setter), 资源提供 者(resource provider), 学习促进者(promoter、困难帮助者(facilitator)、成效评估者(evaluator)。

在学生的自主学习中, 教师应对学生的学习起引导作用。教师要向学生明确概念: “自主 学习” 与 “自己学习” 是完全不同的两个概念。“自主学习” 并非不需要教师的引导。学生 由 “他主” 到 “自主” 也需要一个转换和适应的过程。自主学习需要学生在教师的指导帮助 下独立自主地完成学习任务。在这个学习过程中, 教师需要向学生介绍相关的学习技巧和策 
略并对其进行一定的训练。

教师要根据学生的情况为他们确定学习目标。因受其生理、 年龄、环境等因素的影响, 学生的元认知能力和自我监控能力尚未发展完善, 还不能为自己 的自主学习制定完善的目标和方案, 因此, 教师应该为学生设计自主学习各阶段的学习指标 应该达到的程度, 比如听力理解、阅读能力、词汇掌握、语法水平等。而后, 学生根据教师 制定的总体学习目标和确定的总体学习内容, 制定出符合自己知识积累情况的个性化的自主 学习目标。

教师要为学生的自主学习提供相应的学习资源。虽然传统的教学模式垢病已久, 但是教 师依旧被当作是学生的一种方便有效的资源。随着互联网与现代技术的迅猛发展, 口益增多 的学习资源极为丰富。作为资源引导者, 教师可以给学生介绍一些英语报刊杂志, 比如《英 语沙龙》、(( 21 世纪》和《中国口报》等英语报刊杂志等。也可以是一些不错的外语学习网 站, 例如, 中国口报网、沪江英语网、普特听力网等, 甚至可以推荐VOA, CNN, BBC等英语 本土国家的广播和网站。学生在学习中遇到困难时, 教师应随时准备提供相应信息。

教师是学习促进者。在教学中, 教师最重要也最困难的任务就是组织教学。在中国, 教 育仍然以应试为目标, 这种理念所导向的教学方法阻碍了对学生潜能的开发, 导致一些学生 高分低能的现象。因此, 教师需要把学生从以应试为目标转变成以能力为目标培养模式。教 师不再是一个演讲者, 知识的传送者, 而是一名设计师或者说是学习氛围的创造者。Littlewood 认为学生最想要探求的是知识本身, 专家做过一个实验: “课程一开始, 学生在阅读课文的同 时自学, 并且和同学讨论较难的词组和句子, 然后在黑板上写下那些超出他们理解范围的词 语, 最后全班同学一起讨论。如果他们还无法理解这些难点, 就需要教师来解释。在学生回 答了综合问题之后，他们就有机会向教师提问，这些问题包括一些词语的使用和难句的理解。” 通过这种方法, 我们可以清晰地看到老师从课本的演绎者变成了学习的促进者, 而且课堂中 的难点也在教师的帮助下被克服。根据Nunan的理解, 当传授(教师输出)和接纳(学生输入)加 快时, 学生自主性就得到了加强。这即是说, 教师作为敦促者, 在引导学生如何发挥他们的 自主性, 以及如何促进整个过程等方面, 发挥越来越重要的作用。当学生不确定该怎样采取 行动, 或者下一步该做什么的时候, 教师就应该给予相应的引导或提示。当学生尚未准备好 回答时, 教师要能够给出暗示来引出更多的答案。此外, 教师还可以作为一个 “平等的伙伴” 加入到整个活动过程中, 活动一开始, 教师就需要转换角色, 加入到学生当中去, 并且不再 以权威高高在上的身份出现或支配活动。教师还是学习成效评估者。教师重要的职责之 一就是评价学生的工作, 教师应该关注学生的成功和进步, 所以就要建立一个以成功为目标 的学习氛围。同时学生也会在自主学习方面更加自信。

当然, 在教学中教师要扮演很多的角色。即使是自主学习教学模式中, 主要是以学生为 中心的教学, 但教师仍然扮演着很多重要的角色。除了上述所提到的角色以外, 教师也是学 生自主学习的激发者(motivator)、组织者(organizer)、监控者(monitor)、诊断者(diagnostician)、 学习顾问(counselor)、协调者(negotiator), 个体差异的发现者和指导者(individual difference detector) 。

\section{2. 教师在两种教学模式中的作用}

\section{1教师在 “T-S 教学模式” 中的作用}

" T-S教学模式” 通常被称为引导性教学模式。这种以学生为中心的教学模式是一种与传 统模式不同的师生关系, 强调从过程中学习, 建立一个动态的学习团队, 对学习的过程给予 极大地关注, 其特点是动态教育学的实践原则, 如教师的指导地位, 学生积极参与的作用, 学生的内在动力, 课外学习, 学习过程中认知与情感的同等重要性, 对于学习过程的关注以 及通过自身努力取得学业上的成功。在此模式中, 教师的角色从支配者转换为学生学习活动 中的积极参与者, 与此同时, 教师应设计活动程序, 记录学生的表现, 然后给他们评分和总 
结。这种类型的教学模式改变了以往的传统模式, 注重体验的同时还引入了讨论、交流和探 索的因素。而最为重要的是这种模式主张 “授人以鱼, 不如授人以渔” , 不但解决了学习中 的问题, 还帮助学生掌握了语言应用和创造的能力。教师并不仅仅要给学生知识材料, 而且 还要教给他们获取知识的方法。教师可以通过以下几方面来帮助学生进步。首先, 帮助学生 树立一个明确的目标。教师可以参考大学外语教学大纲, 了解关于听、说、读、写、译的具 体规划, 学生可以制作符合自己需求的计划。其次, 好的学习习惯对学生来说也是至关重要 的, 教师应该指导学生管理他们的学习, 给予他们学习的技巧和策略。一个良好的推动力和 信心能保证T-S教学模式的成功。动机是影响二语习得的其中一个重要因素。学生的学习动机 直接影响他们的学习积极性, 拥有较好动机和较强自信心, 同时又较少被焦虑和脆弱情感缺 陷影响的学生往往在学习中获益匪浅, 反之亦然。教师要掌握学生的心理, 为学生创造动力 与树立信心。在传统的灌输式教学中, 教师是主体和中心, 启发式教学是以学生为中心的, 学生的积极性而学生则是被动的接受者。此外, " T-S 教学模式” 重视师生互动交流。频繁的 师生互动交流是积极有利的学习环境与成功的学习团队的标志。积极有利地学习环境不仅仅 是对学生的课堂学习有效, 对课外学习也是如此。结构主义认为知识的习得取决于学习者本 身, 而不是来源于他人的传授。外语教学的主要任务是帮助学生获得学习自主和语言应用的 能力, 这就要求我们在课堂上采取以学生为中心的教学模式。课堂应该是一个交流的地方, 教师作为引导者, 要带领学生, 并为学生的实践创造机会, 以便开发他们对英语的兴趣。大 学英语和高中英语不同, 单词记忆、语法练习和听写已经不再是学习的目的, 而更多的是强 调听、说、读、写方面的发展, 因此, 教师要让学生明白:仅仅在课堂上的教学是远远不够的。 学习英语的最佳方式和态度就是学生自主, 也就是说, 学生应该自行调配他们的课外学习。对 于 “T-S 教学模式” 来说, 学习策略的总结是必要的。为了确保T-S教学模式的效用, 学生要 负责他们自身的课余活动, 教师也要了解其自主学习的过程, 并为学生总结和比对有效的方 式和方法, 让学生对成效有直观的体验和感受, 在改进自主学习中也会起到良性作用。

\section{2教师在 “S-S 教学模式” 中的作用}

"S-S教学模式” 在学生自主学习中被称为协作学习。学生被分成许多协作组, 然后配合 团队完成任务。同学间的互教活动, 相互评定, 自我评价, 团队协作, 同一团队内的共同目 标, 资源共享, 这对学生的自主学习有着积极地影响。对于学生自主学习的训练有如下特点: 协商沟通, 以过程为中心, 积极地相互依靠。老师与学生共同商量如何组织教与学的过程、 选择学习内容以及如何对学习结果做出评价。 Boekartes认为协作学习是以目标为中心的 是独特的。这也是学生自主学习训练的特点, 因为每个学生的表现与想法都是受欢迎的, 在 学习的不同阶段都可以体现出学生们的不同目标。关于积极地相互依靠, 作为一个学习团队 的一员, 学生明白团结力量大。团队的成功也就是组员个人的成功, 反之亦然, 但是, 这

些需要学生之间的相互依靠。学业上的成功在很大程度上取决于学生们是否在课堂内外 选择了相关适当的语言内容。成功还取决于学生们是否以一种合适的方式将所学展示出来, 还有他们是否认真的相互评价, 给同伴有用的反馈信息。这一点与Cohen提出的资源共享一致, 因为同伴就是实践与评价的来源。每一个学生都对他同伴的成功起着积极地作用。从这个层 面上说, 为了达到小组的目标, 学生们应该彼此依靠, 这就是所谓的目标互相依赖原则。在 “s-s教学模式” 中, 老师的作用不仅仅是传统意义上的传播知识, 更应该是促进学生能力的 提高。老师有着高度的自主能力, 他们非常灵活, 可以持续学习的负责任的人。在教室里, 他们即使是把权力交到学生的手里也不会失去权威, 他们不断地实践与学习的同时, 也为学 生树立的很好的榜样。教师的作用主要从两个方面表现出来。在 “s-s教学模式” 中, 老 师的作用不仅仅是传统意义上的传播知识, 更应该是促进学生能力的提高。老师有着高度的 自主能力, 他们非常灵活, 可以持续学习的负责任的人。在教室里, 他们即使是把权力交到 学生的手里也不会失去权威, 他们不断地实践与学习的同时, 也为学生树立的很好的榜样。 教师的作用主要从两个方面表现出来。 一个方面是制定协作组的分配原则，另一个方面 
是为每个协作组建立相同的目标和任务。通常, 不同班级学生的人数不同, 学生的外语水平 也各异, 教师要把各种因素均考虑在内, 使优等生, 中等生平均分布在每一个协作组里, 这 样，后者就可以从前者那里获得更好的语言输入知识，同时，前者也有更多的机会来 输出自己的见解。这样的协作组让每个人都得到各自想要的东西。

在为每个协作组建立相同的目标和任务时，教师可以从他的教学实践中获得进步。教师 可以尝试指导学生, 并且有可能借助提问来吸引学生自觉反映他们自身的学习状况, 这样对 于提高学生的学习意识是很有效的, 而且, 教师也可以从事各种各样的研究活动。在教学过 程中, 反映性教学被并入学生自主学习的开发中。教师同时参与教和学两个过程, 因此在专 业建设方面促进了教师自主。在以学生为中心的方法中, 教师的角色被重新定义了, 他 被赋予了辅助者、总结者和学习资源的管理者等角色。他们作为辅助者, 通过提升学生的觉 悟来为学习提供支持, 帮助学生设定学习计划, 监督他们的学习过程, 评估他们的学习成果, 促进他们语言能力。作为指导者, 教师的作用应该是为学生提供指导与意见, 教师与学生应 该有一对一的交流; 作为学生管理者的作用指的是教师是知识与专业技能的来源, 为那些自主 学习的学生提供已有的信息。教师应该为学生提供一个唤醒学习动力从而使学生能自主学习 的学习环境。因此, 在学生进行自主学习的过程中, 教师的作用已经不仅仅是促进者, 指导 者以及学习的管理者, 还要制定策略使学生成为高效独立的自主学习者。教师在培养学 生自主学习中很重要的一个环节是课外独立练习。课外独立练习重要的是要建立一种关系, 一种可以公开批评和商讨学习内容和目标的关系, 学生课外独立练习只有在对学习成功反复 思考的情况下才是有效地。对学生的培训要充分利用每个学生的特点以及他们以往学到的知 识。靠规定无法让学生自主学习, 实现自我管理的, 应该让自主学习建立在学生的思考之上。 应该帮助每个学生找到适合他们自己的学习方法。

\section{3. 培养学生的自主学习意识的方法}

\section{1 问卷调查}

教师可以就学生的学习目标以及学习策略展开问卷调查, 每学期两到三次。这样既对教 师更好的了解学生有益, 也可以让学生明白自己的自主学习状况。

\section{2 深度访谈}

与学生进行深度访谈可以以一种更直接更开放的方式帮助学生更好的了解他们自身的英 语学习。教师可以通过问学生如关于英语学习与教学的态度, 学习中的需要, 学生与老师的 作用, 学习中的困难以及应对的策略的问题与学生互动。

\section{3学生日记}

学生的口记是学生做的关于自己语言学习经验和对学习过程反思的能反映问题的第一手 资料。Nunan发现在指导下的思考非常有效。“三省吾身” 的教育理念适用于每一个懂得思 考, 并从思考中纠正自己错误的学习者。这样的进步往往是最大的。

\section{4. 正确看待教师在学生自主学习中的作用}

没有自主的教师很难有自主的学习。自主的教师是独立的, 有着自给自足的个性, 他们 把教学当作是自己职业道德方面的责任。自主的教师们在教室中的主要作用不是传授知识。 相反的, 他们表现为组织者, 建议者以及信息的来源。教师的作用以及学生的自主学习是相 互关联的。没有教师, 学生的自主学习不能完成。教师们要能够将传统的以教师为中心的模 式转变为以学生为中心的模式以帮助他们成为可以自主学习的学习者, 教师们还需要让自己 去适应如下的新角色, 如促进者和咨询者, 通过这些角色, 教师们可以为学生的自主学习创 
造环境, 培养他们的意识最终使他们可以自主学习。教师应该调整自己的学习和授课态度, 把很多传统的教师的角色转给学生, 让学生越来越少地依靠教师, 自己满足自己的学习需求。 教师更多的是资源的发现者、提供咨询者、学习促进者而不是教室中的裁决者或者掌权者。 学生应该把 “学习者” 这个身份记在心里, 而不是总是想向教师求助。教师还应当在培养学 生自主学习能力的同时培养自己处理学生在学习过程中的焦虑情绪的能力, 因为学生学习的 的失败也就意味着教师教学的失败。教师以积极和客观的态度面对国家性和地区性的教育改 革, 引导学生建立终身的学习意识, 把建设学生自主当做职责所在, 把专业发展融入到教学 实践中去。灵活处理外部和内部约束使教学和学习受益, 帮助学生建立自主学习的意识、获 得自主学习的能力, 以助其今后的终身学习。

\section{References}

[1] Fan Jie Ping. Foreign language learning and creative talents training in research universities $\{\mathrm{J}\}$. foreign language and foreign language teaching,2004 (6): 19-21.

[2] Fan Ye. On "learner training" in College English teaching $\{\mathrm{J}\}$. foreign language field, 1999 (3): $32-37$.

[3] Ma Rui. On the influence of humanistic learning theory on modern teaching technology $\{\mathrm{J}\}$. Chinese audio-visual education, 1996 (10).

[4] Ma Xiu Qi and Diao Xiuli. The value and application of modern humanistic education theory, $\{\mathrm{J}\}$. audio-visual education research, $2002(9>)$.

[5] Gao Geely. An overview of foreign language learning in China. $\{\mathrm{J}\}$. foreign language teaching, 2005 (1): 60-63.

[6] Gao Peng and Zhang Xuezhong. The cultivation of learner autonomy in College English classroom $\{J\}$. foreign language community, 2005 (1): 39-45.

[7] Hua Wei Fei. On the orientation of foreign language teachers in the white dominated learning mode $\{\mathrm{J}\}$. foreign language studies, 2001 (3): 76-79.

[8] A new type of language learning environment, $\{\mathrm{J}\}$., foreign language, 2001 (5): 43-48.

[9] Hua Wei Fan. Strategy training for foreign language learners in \{\} $\mathrm{J}\}$. foreign language field, $2002(3>$ : 2-7.)

[10]Hua Wei Fang. Report on the establishment of an English Autonomous Learning Center \{\}$J\}$. foreign language, 2003 (6): 43-48.

[11]Huang Jing, Phil Benson. Second Language Education Teacher Autonomy Research $\{\mathrm{J}\}$. foreign language and foreign language teaching, 2007 (12): 